\title{
How to See Invisible Universes
}

\author{
Alexander Alexandrovich Antonov \\ Independent Researcher, Kiev, Ukraine \\ Email: telan@bk.ru
}

How to cite this paper: Antonov, A.A. (2020) How to See Invisible Universes. Journal of Modern Physics, 11, 593-607. https://doi.org/10.4236/jmp.2020.115039

Received: March 14, 2020

Accepted: April 27, 2020

Published: April 30, 2020

Copyright $\odot 2020$ by author(s) and Scientific Research Publishing Inc. This work is licensed under the Creative Commons Attribution International License (CC BY 4.0).

http://creativecommons.org/licenses/by/4.0/ (c) (i) Open Access

\begin{abstract}
The article shows that the special theory of relativity (STR) was not actually created in the 20th century, since: 1) the relativistic formulas presented therein are incorrect; 2) the relativistic formulas presented therein are explained incorrectly using the incorrect principle of light speed non-exceedance refuted in the article; 3) the relativistic formulas presented therein rise to incorrect conclusions about physical unreality of imaginary numbers and existence of only our visible universe. Moreover, the STR could not even have been created in the 20th century since: 1) experimental data on the six-dimensional space of our hidden Multiverse, which allowed to derive the correct relativistic formulas, were obtained by the WMAP and Planck spacecraft only in the 21 st century; 2) the principle of physical reality of imaginary numbers, which allowed to refute the principle of not exceeding the speed of light and correctly explain the new relativistic formulas, was experimentally proved only in the 21st century. Therefore, the new relativistic formulas obtained given these circumstances allow to state that, in addition to our visible universe, there are numerous other mutually invisible universes, which together form the hidden Multiverse. Existence of invisible universes explains the phenomenon of dark matter and dark energy. Their existence also explains why antimatter in the hidden Multiverse does not annihilate with matter, and tachyons do not violate the principle of causality. The existence of these invisible universes can be proved by astronomical observations in the portals, since in them the constellations of the starry sky will differ from the constellations observed from existing observatories on Earth.
\end{abstract}

\section{Keywords}

Imaginary Numbers, Special Theory of Relativity, Dark Matter, Dark Energy, Dark Space, Multiverse, Hyperverse, Invisible Universes, Portals 


\section{Introduction}

To see invisible universes means to ascertain their real physical existence and, therefore, discover them. Moreover, this means their indisputable discovery, since the universes can be surely identified by the constellations visible in their sky, like people can be identified by fingerprints. That is, constellations are a kind of passport of any universe that cannot be forged.

To see invisible universes, one needs to know how to do this, as they have not yet been discovered. However, in the existing version of the special theory of relativity (STR) [1] [2] [3] it is stated that in accordance with the principle of not exceeding the speed of light, other universes, except our visible universe, do not exist in nature. And this statement seems to have been even confirmed experimentally, as astronomers have never seen any other constellations in the sky, except those visible always. It is even considered that all the hypotheses of the Multiverse, proposed by scientists, some of which are given in [4]-[10], are unverifiable, i.e. they will neither now, nor in the distant future be in any way discovered by the inhabitants of the Earth.

So why then did the question referred to in the article headline arise? This is because other universes actually exist and can be seen by people [11].

\section{Proof of Physical Reality of Concrete Imaginary Numbers}

However, attempts to see invisible universes would make sense only provided that there is confidence in their existence and possibility of detection; that is, provided that it will be understood how this could be done. Then it would be proved that the STR denying this possibility and thereby the existence of Multiverses is wrong.

In order to make sure that the STR is wrong, we should recall that the principle of light speed non-exceedance implies not only denial of existence of Multiverses, but also denial of physical reality of imaginary numbers discovered five hundred years ago. And this turned out to be the whole point, since the authors of the STR did not know how to explain the relativistic formulas [12], in which all physical quantities, such as mass, time, etc. became imaginary at superluminal speeds. Neither the authors of the STR nor anyone before them knew what is, for example, $5 i$ kilograms, $3 i$ seconds, $2 i$ meters, where $i=\sqrt{-1}$. However, physicists and mathematicians who lived before creation of the STR did not assert that imaginary numbers were not physically real. They admitted that they had no answer to this question. In contrast, the authors of the STR didn't get to admit this, since otherwise the STR would not have received recognition. That's why the STR turned out to require the postulate (i.e. the unproven assumption) on light speed non-exceedance, as it allowed the physical nature of concrete imaginary numbers not to explain.

However, Nobel Prize winner Stephen Weinberg was pretty clearly about such theories based on postulates: "Scientific theories cannot be deduced by purely mathematical reasoning". Therefore, the principle of light speed non-exceedance 
in STR always caused doubt ${ }^{1}$ in physicists. And in the 21 st century physicists decided to verify experimentally the principle of light speed non-exceedance, as well as the assertion of physical unreality of imaginary numbers that followed from it. In 2011, the OPERA collaboration published a sensational report [13] stating that it had managed to refute the principle of light speed non-exceedance experimentally. But six months later the ICARUS collaboration refuted the OPERA experiment [14]. And thus impression was created that the principle of light speed non-exceedance is irrefutable.

However, it is possible that the creation of such an illusion was the true goal of the OPERA and ICARUS experiments. After all, back in 2008-2010 reports [15]-[20] were published on experimental studies of special processes in linear electric circuits, in which the general scientific principle of the physical reality of imaginary numbers was successfully proved. Therefore, the OPERA experiment has become unnecessary. Reports on further studies of the same purpose were published in [21]-[28]. But while the unsuccessful and unnecessary very expensive OPERA experiment was intensively touted, the physical community did not pay attention to simple alternative successful experimental evidence of the physical reality of imaginary numbers. Therefore, the assumption that the purpose of the OPERA and ICARUS experiments was not the search for scientific truth, but some other non scientific interests, does not seem to be unfounded.

Moreover, in publications [15]-[28] even three experimental proofs of the principle of physical reality of imaginary numbers were given:

- using transient oscillatory processes [19] [20] [24] [28], that allowed us to conclude that tsunamis would not have existed, church bells wouldn't have sounded and children's swing pushed by parents wouldn't have swung, if the STR statement about physical unreality of imaginary numbers were true;

- using resonant oscillatory processes [15] [16] [17] [18] [20] [21] [24] [28], that along with television, radiolocation and telecommunication could not have existed, if the STR statement about physical unreality of imaginary numbers were true;

- using Ohm's law in the interpretation of Steinmets [22] [23] [25] [26] [27] [28], proposed by him in 1893, which made it possible to refute the existing version of STR even before its creation.

And since the experiments presented in these publications, unlike the OPERA and ICARUS experiments, are simple and can be repeated in any radio engineering laboratory, the principle of physical reality of imaginary numbers is

- ${ }^{1}$ The STR has been criticized by Oliver Heaviside, Nikola Tesla, Nobel Prize winner Albert Abraham Michelson, Nobel Prize winner Friedrich Wilhelm Ostwald, Nobel Prize winner Joseph John Thomson, Nobel Prize winner Svante August Arrhenius, Nobel Prize winner Philipp Eduard Anton von Lenard, Nobel Prize winner Alvar Gullstrand, Nobel Prize winner Wilhelm Carl Werner Otto Fritz Franz Wien, Nobel Prize winner Walther Hermann Nernst, Nobel Prize winner Ernest Rutherford, 1st Baron Rutherford of Nelson, Nobel Prize winner Johannes Stark, Nobel Prize winner Frederick Soddy, Nobel Prize winner Percy Williams Bridgman, Nobel Prize winner Edwin Mattison McMillan, Nobel Prize winner Hideki Yukawa, Nobel Prize winner Hannes Olof Gösta Alfven and many other outstanding scientists. 
indisputably proved by these experiments. The reluctance of the physical community to take into account the successful evidence of the physical reality of imaginary numbers set forth in these alternative radio engineering experiments speaks of the shortcomings in modern physical education.

Moreover, the denial in STO of the physical reality of the concrete imaginary numbers is equivalent to the denial of the physical reality corresponding to these numbers of the invisible and therefore still largely unknown world ${ }^{3}$ which science will know about in the future. And this inhibits the development of science. Noting the great importance of imaginary numbers in the science, Sir Roger Penrose wrote: "The system of complex numbers has a profound and timeless reality which goes beyond the mental constructions of any particular mathematician ... They were put there neither by Cardano, nor by Bombelly, nor Wallis, nor Coates, nor Euler, nor Wessel, nor Gauss, despite the undoubted farsightedness of these, and other, great mathematicians, such set of magical properties was inherent in the very structure that they gradually uncovered ..."

Therefore, taking into account the principle of physical reality of imaginary numbers, although unacknowledged in physics, but experimentally proved in radio engineering, all physical theories and hypotheses should now be corrected.

\section{Explanation of Physical Nature of Concrete Imaginary Numbers in the STR}

Sometimes it is written that the STR has refuted the classical physics of Galileo, Newton and other great scientists of the past. But it is not so. In fact, as can be seen from Table 1 presenting the results of calculations using relativistic formulas, for example,

$$
\begin{gathered}
m=\frac{m_{0}}{\sqrt{1-(v / c)^{2}}} \\
\Delta t=\Delta t_{0} \sqrt{1-(v / c)^{2}}
\end{gathered}
$$

where $m_{0}$ is the rest mass of a physical body;

$m$ is the relativistic mass of a moving physical body;

$\Delta t_{0}$ is the rest time of a physical body;

$\Delta t$ is the relativistic time of a moving physical body;

$v$ is the velocity of a physical body;

$c$ is the speed of light,

Relativistic effects become noticeable only at speeds comparable to the speed of light. But such speeds are found only in space or at subatomic particle accelerators. There are no such speeds in everyday life, like in transport, in industry or in natural processes on Earth.

${ }^{2}$ What evidence could be more convincing than existence of, for example, TV-sets, tsunamis or children's swings that should not have existed, if the STR statement about physical unreality of imaginary numbers were true?

${ }^{3}$ However, an invisible world exists. For example, from the room in which we are now, the next room is not visible and, therefore, at that time it is an invisible world. Nevertheless, no one will deny its existence. 
Table 1. Calculations, illustrating the absence of a relativistic effect in everyday human life in transport, in industry, in natural processes on Earth.

\begin{tabular}{ccccccc}
\hline$v / c$ & 0.0100 & 0.1000 & 0.9000 & 0.9900 & 0.9990 & 0.9999 \\
$m / m_{0}$ & 1.00005 & 1.00504 & 2.29416 & 7.08881 & 22.36627 & 70.71245 \\
$\Delta t / \Delta t_{0}$ & 0.99995 & 0.99498 & 0.43589 & 0.14167 & 0.04471 & 0.01414 \\
\hline
\end{tabular}

Consequently, the existing version of SRT is not only not quite true, but it is not needed by people in everyday life. And still need classical physics.

But in science, for example, in astrophysics, STR is needed. More precisely, we need a corrected version of it. The denial by the existing version of the SRT of the principle of physical reality of imaginary numbers, as has just been shown, is its mistake. Therefore, the error must be corrected, i.e. it must be taken into account that relativistic formulas (1), (2), etc. must be explainable both in the range of $0 \leq v<c$ and in the range of $c \leq v<\infty$. However, the graphs of formulas (1) and (2) in Figure 1(a), Figure 1(b) shows that the formulas are incorrect, since their graphs in the ranges $0 \leq v<c$ and $c \leq v<\infty$ are of significantly different forms. Therefore, they are inexplicable.

Analysis of their possible explainable options has showed that the graphs of relativistic formulas should be written as shown in Figure 1(c), Figure 1(d). Therefore corrected relativistic formulas should take the following form

$$
\begin{gathered}
m=\frac{m_{0} i^{q}}{\sqrt{1-(v / c-q)^{2}}}=\frac{m_{0} i^{q}}{\sqrt{1-(w / c)^{2}}} \\
\Delta t=\Delta t_{0} i^{q} \sqrt{1-(v / c-q)^{2}}=\Delta t_{0} i^{q} \sqrt{1-(w / c)^{2}}
\end{gathered}
$$

where $q=\lfloor v / c\rfloor$ is the "floor" function of argument $v / c$, whose values ${ }^{4}$

correspond to different parallel ${ }^{5}$ universes;

$w=v-q c$ is the local velocity for each universe, which can take values only in the range of $0 \leq w<c$;

$v$ is the velocity measured for our visible universe.

Thus, formulas (3) and (4) can already be interpreted in a comprehensible manner. Their $q$ parameter can be considered as an extra spatial dimension ${ }^{6}$. Thus, the value $q=0$ would correspond to our visible universe (as $i^{0}=1$ ); and the value $q=1$ would correspond to another universe (as $i^{1}=i$ ) invisible for us, given that $v>c$, as it is beyond the horizon of events. It shall, therefore, be referred to as a tachyon universe, just like subatomic particles moving at superluminal speeds. For the same reasons, our universe shall be referred to as tardyon. The value $q=2$ would correspond to a tardyon antiverse (as $i^{2}=-1$ ), the value $q=3$ would correspond to a tachyon antiverse (as $i^{3}=-i$ ), the value $q=4$ would correspond to another ${ }^{7}$ tardyon universe (as $i^{4}=1$ ), the value $q=5$ would correspond to another tachyon universe (as $i^{5}=i$ for it), etc.

${ }^{4}$ And it takes non-integral values in the portals considered below, in which, their value q changes for one from entry to exit under the influence of physical factors not yet studied.

${ }^{5}$ Called so because they do not intersect, despite their infinity.

${ }^{6}$ Like numbers of apartments in an apartment building.

${ }^{7}$ There are about twenty such invisible universes in the hidden Multiverse, as shown below. 


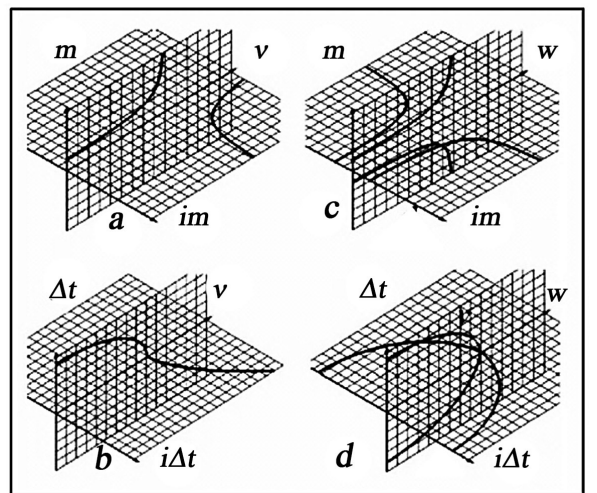

Figure 1. Graphs of functions (1), (2) corresponding to the existing wrong version of the STR, and (3), (4) corresponding to its corrected version.

Thus, concrete imaginary numbers in the corrected relativistic formulas (3), (4), etc. correspond to mutually invisible parallel universes, together forming the Multiverse, which we therefore call the hidden Multiverse [29] [30] [31].

However, the invisible universes mentioned are not a gift in science, since they are known even less than, for example, dark matter and dark energy. Moreover, despite the logically unimpeachable arguments presented in [32] [33] confirming real physical existence of invisible universes, there is still $\mathrm{no}^{8}$ complete certainty about this due to the lack of indisputably conclusive experiments.

\section{Explanation of Dark Matter and Dark Energy by Existence of Invisible Universes of the Hidden Multiverse}

However, existence of dark matter and dark energy that are as much incomprehensible as invisible universes has already been experimentally proven: dark matter in 1932-33 by Jan Hendrick Oort and Fritz Zwicky, and dark energy in 1998-99 by Saul Perlmutter, Brian Schmidt and Adam Riess, who were awarded the Nobel Prize for this discovery. Emphasizing the outstanding importance of these discoveries, Nobel Prize winner Adam Riess wrote: "Humanity is on the verge of a new physics of the Universe. Whether we want it or not, we will have to accept it." And about this new physics in the article is discussed below.

Dark matter and dark energy [34] [35] [36] [37] are called dark for their obscurity. It is unclear why they are absolutely invisible in all ranges of electromagnetic oscillations and can only be detected indirectly by their gravitational manifestations. It is unclear why neither molecules, nor atoms, nor subatomic particles are found in dark matter and dark energy, though their total mass/energy is more than 20 times greater than the mass/energy of our visible universe. Many other things are as well unclear. Therefore, their knowledge in modern physics is a task of paramount importance.

Professor Michio Kaku commented on this situation as follows: "Of course, a whole bunch of Nobel Prizes is waiting for the scientists who can reveal the se${ }^{8}$ However, it is written below how to get them. 
crets of the dark energy and dark matter".

The phenomenon of dark matter and dark energy is currently investigated very intensively. But it is still far from being fully explained, since all the studies are conducted only in terms of the hypothesis of the visible Monoverse that follows from the erroneous version of the STR. And in this case we cannot but agree with the Nobel Prize laureate Albert Einstein, who argued: "Insanity. doing the same thing over and over again and expecting different results". In this regard, it is pertinent to recall Sir Isaac Newton's opinion: "No great discovery was ever made without a bold guess".

Therefore, assuming that there is a hidden Multiverse rather than a Monoverse, the phenomenon of dark matter and dark energy can be explained as follows [38]-[45]:

- dark matter and dark energy do not actually have any real physical contents; they are some kind of image (but not electromagnetic) analogous to an optical, something like a shadow evoked by existence of invisible parallel universes;

- herewith, the phenomenon of dark matter is evoked by invisible parallel universes adjacent to our visible universe, and the phenomenon of dark energy is evoked by other invisible parallel universes of the hidden Multiverse;

- therefore, no material contents, such as molecules, atoms or subatomic particles, will ever be found in dark matter and dark energy;

- dark matter and dark energy are invisible because other parallel universes of the hidden Multiverse, except our visible universe, are invisible.

Such approach allows getting an idea of the structure of the hidden Multiverse, based on experimental data from WMAP spacecraft that was launched by the National Aeronautics and Space Administration (NASA) in 2001 and operated until 2009, as well as data from the Planck spacecraft that was launched by the European Space Agency (ESA) in 2009 and operated until 2013. Thus, according to the WMAP spacecraft date [46], the entire universe (in fact, the entire hidden Multiverse) is $4.6 \%$ composed of baryonic matter, $22.4 \%$ of dark matter, and $73.0 \%$ of dark energy. And according to later data from the Planck spacecraft [47], the entire universe (in fact, the entire hidden Multiverse) is 4.9\% composed of baryonic matter, $26.8 \%$ of dark matter, and $68.3 \%$ of dark energy. So, assuming that over billions of years of existence mass/energy of all invisible universes has substantially averaged due to exchange of their material contents through portals ${ }^{9}$, it can be argued that:

- according to the WMAP spacecraft data the hidden Multiverse is formed by $100 \% / 4.6 \%=21.74$ universes, and according to the Planck spacecraft data they are $100 \% / 4.9 \%=20.41$ universes, i.e. real number of the universes is supposedly 20 ... 22;

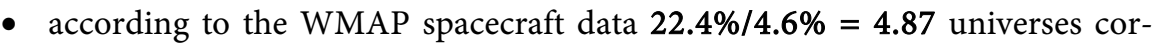
respond to the dark matter phenomenon, and according to the Planck spacecraft data they are $26.8 \% / 4.9 \%=5.47$ universes, i.e. real number of the un-

${ }^{9}$ That will be discussed below. 
iverses is supposedly $5 \ldots 6$;

- according to the WMAP spacecraft data $73.00 \% / 4.6 \%=15.87$ universes correspond to the dark energy phenomenon, and according to the Planck spacecraft data they are $68.3 \% / 4.9 \%=13.94$ universes, i.e. real number of the universes is supposedly $14 \ldots 16$.

And such a result in the 20 th century was impossible to guess by any postulate.

But it is not difficult to notice that the results obtained as a result of calculations do not correspond to the corrected relativistic formulas (3) and (4). Indeed, according to formulas (1) and (2). Our tardyon universe in the hidden Multiverse should have only two adjacent universes in one extra dimension, rather than five or six universes, as follows from the above calculations. Therefore, it follows from the WMAP and Planck spacecraft data, that the number of extra dimensions should be three.

Thus, formulas (3) and (4) must be corrected again as follows

$$
\begin{gathered}
m=\frac{m_{0}\left(i_{1}\right)^{q-q_{0}}\left(i_{2}\right)^{r-r_{0}}\left(i_{3}\right)^{s-s_{0}}}{\sqrt{1-\left[v / c-\left(q+r+s-q_{0}-r_{0}-s_{0}\right)\right]^{2}}} \\
=\frac{m_{0}\left(i_{1}\right)^{q-q_{0}}\left(i_{2}\right)^{r-r_{0}}\left(i_{3}\right)^{s-s_{0}}}{\sqrt{1-(w / c)^{2}}} \\
\Delta t=\Delta t_{0}\left(i_{1}\right)^{q-q_{0}}\left(i_{2}\right)^{r-r_{0}}\left(i_{3}\right)^{s-s_{0}} \sqrt{1-\left[v / c-\left(q+r+s-q_{0}-r_{0}-s_{0}\right)\right]^{2}} \\
=\Delta t_{0}\left(i_{1}\right)^{q-q_{0}}\left(i_{2}\right)^{r-r_{0}}\left(i_{3}\right)^{s-s_{0}} \sqrt{1-(w / c)^{2}}
\end{gathered}
$$

where $q_{0}, r_{0}, s_{0}$ are the coordinates of our visible universe in the hidden Multi-verse:

$v$ is the velocity measured from our tardyon universe;

$c$ is the speed of light;

$w=v-\left(q+r+s-q_{0}-r_{0}-s_{0}\right) c$ is the local velocity for the universe corresponding to the coordinates $q, r, s$ of the universe, which can take values only in the range of $0 \leq w \leq c$.

Herewith, three imaginary units $i_{1}, i_{2}, i_{3}$ that correspond to three extra dimensions and are present in hypercomplex numbers called quaternions [48], are interconnected by the relations

$$
\begin{gathered}
i_{1}^{2}=i_{2}^{2}=i_{3}^{2}=1 \\
i_{1} i_{2} i_{3}=i_{2} i_{3} i_{1}=i_{3} i_{1} i_{2}=-1 \\
i_{1} i_{3} i_{2}=i_{2} i_{1} i_{3}=i_{3} i_{2} i_{1}=1
\end{gathered}
$$

Consequently, the structure of the six-dimensional space (see Figure 2) of the hidden Multiverse can be described by the formula

$f_{q, r, s}(x, y, z)+i_{1} q+i_{2} r+i_{3} s$ in which the summand $i_{1} q+i_{2} r+i_{3} s$ determines the coordinates of the corresponding invisible universe, and the summand $f_{q, r, s}(x, y, z)$ determines the distribution in the coordinates $x, y, z$ of its material contents in this universe. 


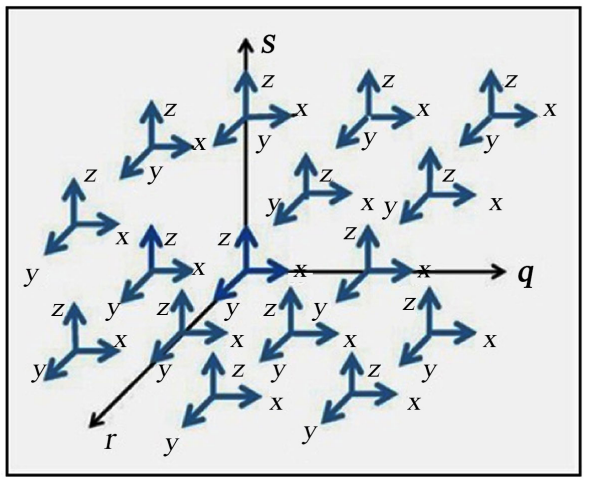

Figure 2. Six-dimensional space of the hidden Multiverse.

Professor Lisa Randall wrote about a similar situation: "We probably live in a three-dimensional gap of higher dimensional space". And her assumption, as seen, was confirmed.

Figure 3 presents an example of such a quaternion structure of the hidden Multiverse corresponding to the results of mathematical processing of WMAP and Planck data and containing twenty-one parallel universes. As can be seen, tardyon universes and antiverses alternate with tachyon universes and antiverses in such an open screw structure. Pursuant to the formula (7), they are interconnected by numerous bidirectional portals denoted by bidirectional arrows. And tachyon universes and antiverses are interconnected by numerous unidirectional portals denoted by unidirectional arrows pursuant to the formulas (8) and (9). Herewith, invisible tachyon universes and antiverses adjacent to our tardyon universe evoke the phenomenon of dark matter. And the remaining invisible universes of the hidden Multiverse evoke the phenomenon of dark energy.

Thus, two obscure concepts "invisible universes" and "dark matter and dark energy" let us understand their meaning in joint consideration. Dark matter and dark energy turn out to be the result of recording gravitational manifestations of invisible parallel universes of the hidden Multiverse in our visible universe.

Advantage of such hypothesis of dark matter and dark energy is that, in addition to explanation of dark matter and dark energy inexplicable until recently, it also explains the inexplicable issues regarding the existence of antimatter and tachyons [49]. As can be seen from Figure 3, antimatter exists in antiverses and it does not annihilate with matter because tardyon universes and antiverses alternate with tachyon universes and antiverses. Moreover, there are even several tardyon and tachyon matters and antimatters in the hidden Multiverse. And tachyons that do not violate the principle of causality exist in tachyon rather than in tardyon universes and antiverses.

\section{Comments on the Existing Version of the STR}

The results obtained are so important that they need additional comments. STR actually consists of two large sections, in the first of which relativistic formulas are derived, and in the second these formulas are explained. 


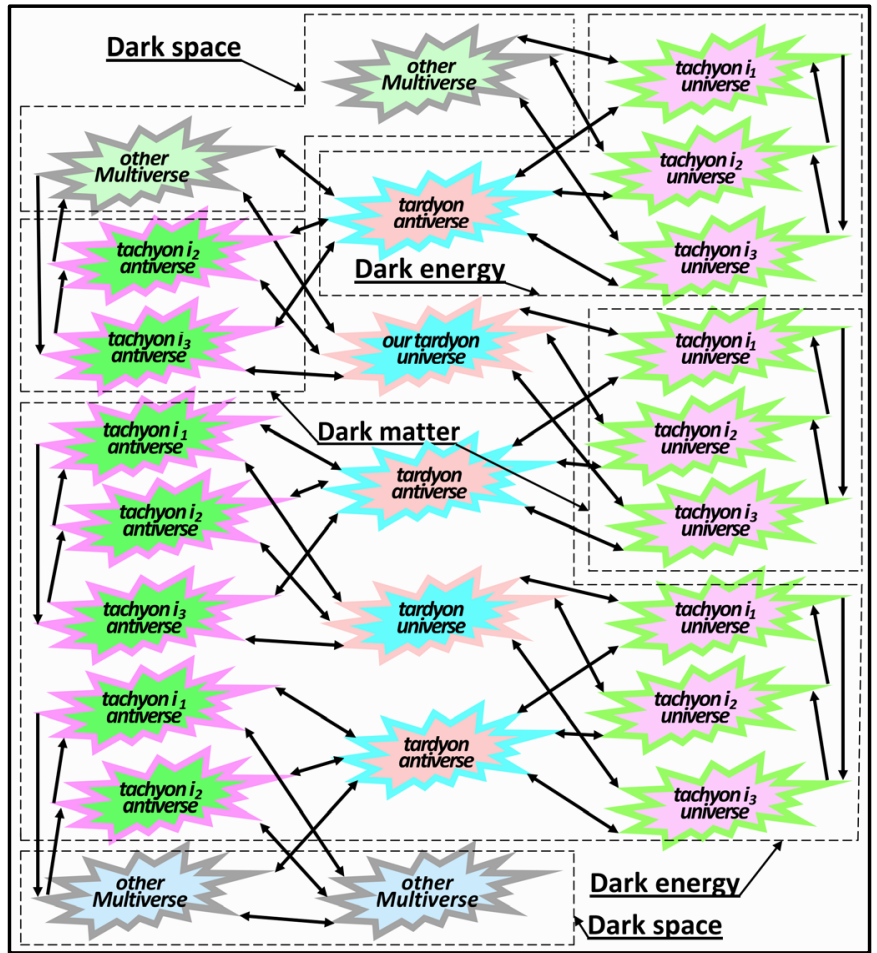

Figure 3. Example of the quaternion structure of the hidden Multiverse.

And it turned out that in both sections of the existing version of the STR, incorrect results were obtained:

- in the first section, instead of the valid relativistic formulas (5) and (6), the incorrect formulas (1) and (2) are obtained;

- in the second section, from the wrong relativistic formulas (1) and (2), erroneous conclusions were drawn about the physical unreality of imaginary numbers and the existence in nature of our only visible universe.

Thus, since the existing version of the STR is incorrect, we have to conclude that in the 20th century STR was not created [50] [51]. Moreover, it could not be created at that time, since Albert Einstein was ahead of his time the experimental data necessary for the derivation of relativistic formulas (5), (6) and other spacecraft WMAP and Planck were obtained only in the 21st century. And the principle of the physical reality of imaginary numbers, necessary for the interpretation of these formulas, was also proved only in the 21st century.

Nevertheless, the very statement of the task of creating STR is an outstanding scientific achievement of Albert Einstein. Without an intermediate result in the form of formulas (1), (2), the final result in the form of formulas (5), (6) would not have been obtained. And the basic principles for solving this problem are described in this article.

\section{Explanation of Dark Space by Existence of Invisible Universes of the Hyperverse}

As can be seen from Figure 3, our visible universe and some invisible universes 
in the hidden Multiverse are connected not only with each other but also with invisible universes of other Multiverses through unidirectional and bidirectional portals denoted by single-sided and double-sided arrows [52] [53] [54]. These other Multiverses relevant to the phenomenon of dark space [55] [56] together with our hidden Multiverse form the Hyperverse (see Figure 4).

And if this were not so, then the hidden Multiverse would have to have edges that contradict common sense and cannot be explained in any way, as, for example, archaic conception about shape of Earth.

But the phenomenon of dark space with WMAP and Planck spacecraft is not registered. However, it can be registered with the studies described below of invisible universes in portals.

\section{Verifiability of Invisible Universes}

Now it's time to answer the question formulated in the article headline "How to see invisible universes?" The question can also be formulated in another way, since it is not completely clear what means to see a universe and why it is necessarily.

This is not necessarily, but such evidence of existence of invisible universes is the most understandable and convincing. Invisible universes can be compared with rooms of our house that are invisible to us from the room we are in now. It is clear that to see invisible rooms one has to come to the doors and look into or enter the rooms. In the same way, to see invisible universes, one has to penetrate the portals and look at the starry sky. It must be different in other universes. Other constellations must be observed on it [11] [55] [56]. And these differences would apparently be the greater the further one enters the portals by foot or by vehicle ${ }^{10}$. The starry sky would be completely different after leaving portals and getting into other universes, which would become visible to us, unlike our universe.

Besides, it is clear that satisfaction of our curiosity in this way would be complicated by two things. The first is that the portals are invisible, and the second is that we haven't been invited to other universes through these portals. However it is possible that some portals, which are very numerous on the Earth [57], are not protected from our visit. Therefore, one can probably try to visit them.

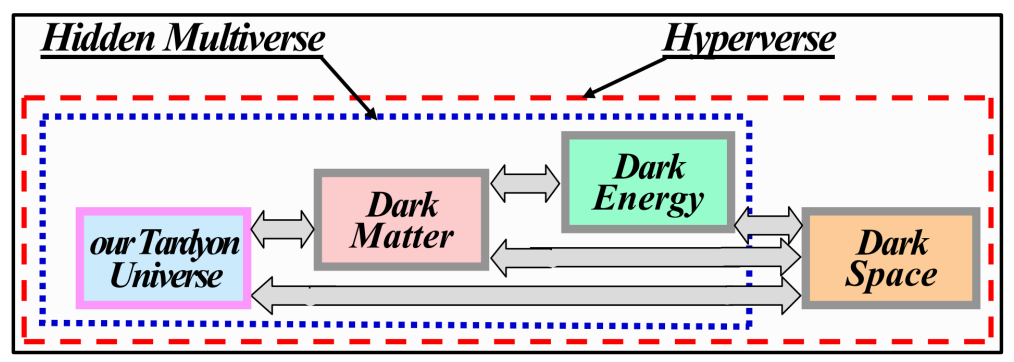

Figure 4. Structure of the Hyperverse.

${ }^{10}$ Therefore, there is no need to fly in portals and through portals by rockets. 
In order not to get lost in the portals and to have a guaranteed opportunity to return to Earth located in our universe after visiting them, it is desirable to have some navigation devices, a kind of a marine compass. These devices of portal navigation should indicate the direction of movement from portal entries to exits and vice versa. Creation of such devices is possible, because with distance from portal entry radio signal strength, e.g, for mobile communication, would decrease and after leaving the portal for the adjacent universe, the signal will completely disappear.

However, it is not necessary to penetrate the portals far off their entries and use navigation devices make certain of existence of invisible universes. The fact is that as you move through the portal from our visible universe to one of the neighboring invisible universes, the map of the earth's starry sky will smoothly transform into a map of the starry sky of another universe. At that the maps of the starry sky in our and in any of the neighboring universes, of course, differ in an extreme way. Therefore, even with shallow penetration into the portals, it will be found that the constellations above the head of the researcher will be so markedly different from the constellations observed in Earth observatories that even amateur astronomers can probably detect and register these changes with their telescopes. And of course, these differences will be noticeably larger than the differences recorded in 1919 in a similar experiment by Sir Arthur Stanley Eddington [58].

\section{Conclusions}

The article shows that the existing version of SRT is incomplete and, therefore, in the 20th century this theory has not yet been created. Moreover, SRT in the 20th century could not have been created, since the data obtained by the WMAP and Planck spacecraft only in the 21 st century were necessary to derive the correct relativistic formulas. And for their correct interpretation, the principle of the physical reality of imaginary numbers was needed, experimentally proved in the 21 st century. The article also proposes and explains the corrected relativistic formulas of the STR, from which it follows that there is a hidden Multiverse formed by invisible parallel universes.

It is explained how the existence of invisible universes can be convincingly proved experimentally. For this, it is enough to use the obvious truth, the starry sky of other universes contains other constellations. Therefore, in portals, which are transitional zones between neighboring universes, the constellations of the starry sky should also be different. And this starry sky in the portals, which on Earth are, at least, some of the many so-called anomalous zones, can be seen and registered. In the entire history of the existence of astronomy, it has not had a task more interesting and more important than the discovery and study of invisible universes.

Thus, the hypothesis of the hidden Multiverse and Hyperverse set forth in the article is verifiable, and the invisible universes are real and can be seen. 


\section{Acknowledgements}

The author is grateful for participation in the discussion of the paper to Olga Ilyinichna Antonova, whose criticism and valuable comments contributed to improvement of the article.

\section{Conflicts of Interest}

The author declares no conflicts of interest regarding the publication of this paper.

\section{References}

[1] Einstein, A. (1920) Relativity: The Special and General Theory. H. Holt and Company, New York.

[2] Bohm, D. (2006) The Special Theory of Relativity. Routledge, Abingdon-on-Thames?'

[3] Hawking, S.W. and Penrose, R. (2010) The Nature of Space and Time. Princeton University Press, Princeton. https://doi.org/10.1515/9781400834747

[4] Kaku, M. (2006) Parallel Worlds: A Journey through Creation, Higher Dimensions, and the Future of the Cosmos. Anchor, New York.

[5] Vilenkin, A. (2007) Many Worlds in One: The Search for Other Universes. Hill and Wong, New York.

[6] Carr, B. (2009) Universe or Multiverse? Cambridge University Press, Cambridge.

[7] Gribbin, J. (2010) In Search of the Multiverse: Parallel Worlds, Hidden Dimensions, and the Ultimate Quest for the Frontiers of Reality. Wiley \& Sons Inc, Hoboken.

[8] Greene, B. (2011) The Hidden Reality: Parallel Universes and the Deep Laws of the Cosmos. Vintage, New York.

[9] Hawking, S. and Mlodinow, L. (2012) The Grand Design. Bantam, New York.

[10] Tegmark, M. (2014) Our Mathematical Universe: My Quest for the Ultimate Nature of Reality. Vintage, New York.

[11] Antonov, A.A. (2019) Journal of Modern Physics, 10, 1006-1028. https://doi.org/10.4236/jmp.2019.108067

[12] Antonov, A.A. (2014) American Journal of Scientific and Industrial Research, 5, 40-52.

[13] Adam, T., Agafonova, N., Aleksandrov, A., et al. (2012) Journal of High Energy Physics, 2012, Article No. 93. https://doi.org/10.1007/JHEP10(2012)093

[14] Antonello, M., Baibussinov, B., Boffelli, F., et al. (2012) Precision Measurement of the Neutrino Velocity with the ICARUS Detector in the CNGS Beam.

[15] Antonov, A.A. (2008) European Journal of Scientific Research, 21, 627-641.

[16] Antonov, A.A. (2009) European Journal of Scientific Research, 28, 193-204.

[17] Antonov, A.A. (2010) American Journal of Scientific and Industrial Research, 1, 342-349. https://doi.org/10.5251/ajsir.2010.1.2.342.349

[18] Antonov, A.A. (2010) International Journal of Pure and Applied Sciences and Technology, 1, 1-12.

[19] Antonov, A.A. (2010) General Mathematics Notes, 1, 11-16.

[20] Antonov, A.A. (2013) Unpredictable Discoveries. Lambert Academic Publishing, Saarbrücken. 
[21] Antonov, A.A. (2015) General Mathematics Notes, 31, 34-53.

[22] Antono, A.A. (2015) American Journal of Electrical and Electronics Engineering, 3, 124-129.

[23] Antonov, A.A. (2015) Global Journal of Physics, 2, 145-149.

[24] Antonov, A.A. (2016) General Mathematics Notes, 35, 40-63.

[25] Antonov, A.A. (2016) PONTE, 72, 131-142. https://doi.org/10.21506/j.ponte.2016.7.9

[26] Antonov, A.A. (2016) Journal of Modern Physics, 7, 2299-2313. https://doi.org/10.4236/jmp.2016.716198

[27] Antonov, A.A. (2016) International Review of Physics, 10, 31-35. https://www.praiseworthyprize.org/jsm/index.php?journal=irephy\&page=article\&o $\mathrm{p}=$ view\&path $\% 5 \mathrm{~B} \% 5 \mathrm{D}=18615$

[28] Antonov, A.A. (2017) Norwegian Journal of Development of the International Science, 6, 50-63.

[29] Antonov, A.A. (2015) International Journal of Advanced Research in Physical Science, 2, 25-32.

[30] Antonov, A.A. (2016) Journal of Modern Physics, 7, 1933-1943. https://doi.org/10.4236/jmp.2016.714170

[31] Antonov, A.A. (2017) Natural Science, 9, 43-62. https://doi.org/10.4236/ns.2017.93005

[32] Antonov, A.A. (2016) Global Journal of Science Frontier Research: A Physics and Space Science, 16, 4-12.

[33] Antonov, A.A. (2017) Global Journal of Science Frontier Research. http://blog.gjsfr.org/2017/02/verifiable-hidden-multiverse.html

[34] Ruiz-Lapuente, P. (2010) Dark Energy: Observational and Theoretical Approaches. Cambridge University Press, Cambridge. https://doi.org/10.1017/CBO9781139193627

[35] Amendola, L. and Tsujikawa, S. (2010) Dark Energy: Theory and Observations. Cambridge University Press, Cambridge. https://doi.org/10.1017/CBO9780511750823

[36] Bertone, G. (2013) Particle Dark Matter: Observations, Models and Searches. Cambridge University Press, Cambridge.

[37] Sanders, R.H. (2014) The Dark Matter Problem: A Historical Perspective. Cambridge University Press, Cambridge.

[38] Antonov, A.A. (2015) American Journal of Modern Physics, 4, 1-9. https://doi.org/10.11648/j.ajmp.20150401.11

[39] Antonov, A.A. (2015) International Journal of Physics, 3, 84-87.

[40] Antonov, A.A. (2015) Cosmology, 19, 40-61.

[41] Antonov, A.A. (2015) American Journal of Modern Physics, 4, 180-188. https://doi.org/10.11648/j.ajmp.20150404.14

[42] Antonov, A.A. (2015) Optics, 4, 43-47.

[43] Antonov, A.A. (2016) Frontiers of Astronomy, Astrophysics and Cosmology, 2, 1-9.

[44] Antonov, A.A. (2016) Journal of Modern Physics, 7, 1228-1246. https://doi.org/10.4236/jmp.2016.710111

[45] Antonov, A.A. (2017) Journal of Modern Physics, 8, 567-582. https://doi.org/10.4236/jmp.2017.84038 
[46] Hinshaw, G., Larson, D., Komatsu, E., et al. (2013) Nine Year Wilkinson Anisotropy Probe (WMAP) Observations: Cosmological Parameter Results.

[47] Adam, R., Ade, P.A.R., Aghanim, N., et al. (2015) Plank 2015 Results. 1. Overviev of Products and Scientific Results.

[48] Kantor, I.L. and Solodovnikov, A.S. (1989) Hypercomplex Numbers. Springer, Verlag, Berlin. https://doi.org/10.1007/978-1-4612-3650-4

[49] Antonov, A.A. (2016) PONTE, 72, 288-300. https://doi.org/10.21506/j.ponte.2016.9.22

[50] Antonov, A.A. (2019) Journal of Russian Physical-Chemical Society, 91, 57-94. (In Russian) http://rusphysics.ru/files/Antonov.91-1.pdf

[51] Antonov, A.A. (2020) Journal of Modern Physics, 11, 324-342. https://doi.org/10.4236/jmp.2020.112020

[52] Antonov, A.A. (2015) Where to Look for Alien Civilisations. Cosmology. Commentaries: Stephen Hawking's Aliens. The Search for Intelligent Extraterrestrial Life. Project Breakthrough Liste. http://cosmology.com/Aliens1.html

[53] Antonov, A.A. (2016) How Portals of the Invisible Multiverse Operate. SciencePG Frontiers. Research Blog. http://www.sciencepublishinggroup.com/news/sciencepgfrontiersinfo?articleid=147

[54] Antonov, A.A. (2016) Philosophy and Cosmology, 6, 11-27. (In Russian)

[55] Antonov, A.A. (2018) Journal of Modern Physics, 9, 14-34. https://doi.org/10.4236/jmp.2018.91002

[56] Antonov, A.A. (2018) Natural Science, 10, 11-30. https://doi.org/10.4236/ns.2018.101002

[57] Chernobrov, V.A. (2000) Encyclopedia of Mysterious Places of the Earth. Veche Publishing, Bucharest. (In Russian)

[58] Dyson, F.W., Eddington, A.S. and Davidson, C. (1920) Philosophical Transactions of the Royal Society A: Mathematical, Physical and Engineering Sciences, 220, 291-333. https://doi.org/10.1098/rsta.1920.0009 